\title{
Formación de Hidrocarburos Aromáticos Policíclicos (PAH) y Hollín en la Pirólisis de 2,5-Dimetilfurano y 2-Metilfurano a Diferentes Temperaturas
}

\author{
Fausto Viteri ${ }^{1,2}$, Pablo Salvo ${ }^{1}$, Cristian Baena ${ }^{1}$, Ángela Millera ${ }^{1}$, Rafael Bilbao ${ }^{1}$, María \\ U. Alzueta $^{1}$ \\ ${ }^{1}$ Grupo de Procesos Termoquímicos (GPT) Instituto de Investigación en Ingeniería de Aragón (I3A). \\ Universidad de Zaragoza, Mariano Esquillor s/n, 50018, Zaragoza, España. \\ Tel. +34-976762707, e-mail: fviteri@unizar.es \\ ${ }^{2}$ Universidad Tecnológica Equinoccial, Quito, Ecuador
}

\begin{abstract}
En el presente trabajo se ha estudiado la influencia de la temperatura en la formación de hidrocarburos aromáticos policíclicos y hollín durante la pirólisis de 2,5-dimetilfurano y 2-metilfurano, los cuales se pueden sintetizar a partir de celulosa biomásica no utilizable en alimentación, y tienen características físicoquímicas que los permiten formar parte de mezclas con gasolina.
\end{abstract}

\section{Introducción}

Se han propuesto varios aditivos como combustibles renovables, por su característica de disminuir la emisión de varios contaminantes atmosféricos. Entre los combustibles propuestos están los compuestos derivados del furano: 2,5-dimetilfurano (2,5-DMF) y 2-metilfurano (2-MF), los cuales han cobrado gran interés debido a nuevas rutas de su síntesis [1], y a que pueden mejorar algunas características de la gasolina [2].

El presente estudio investiga la formación de PAH y hollín a partir de la pirólisis de 2,5-dimetilfurano y 2-metilfurano. Se han analizado 16 hidrocarburos aromáticos policíclicos (PAH), clasificados por la Agencia de Protección Ambiental (EPA) como contaminantes prioritarios debido a su potencial cancerígeno. Los 16 PAH analizados han sido: Naftaleno (NAPH), Acenaftileno (ACNY), Acenafteno (ACN), Fluoreno (FLUO), Fenantreno (PHEN), Antraceno (ANTH), Fluoranteno (FANTH), Pireno (PYR), Benzo(a)antraceno (B(a)A), Criseno (CHR), Benzo(b)fluoranteno (B(b)F), Benzo(k)fluoranteno $\quad(B(k) F)$, Benzo(a)pireno (B(a)P), Dibenzo(a,h)antraceno (DB(ah)A), Benzo(g,h,i)perileno (B(ghi)P), Indeno(1,2,3-c,d)pireno (I(123-cd)P).

\section{Metodología}

Los experimentos se realizan en condiciones de laboratorio controladas. La descripción de las instalaciones se puede encontrar en otros trabajos realizados por nuestro grupo de investigación [3].

Los experimentos de pirólisis de 2,5-DMF y 2-MF se han realizado a tres temperaturas: 1473,1373 y 1273 K, y a presión atmosférica. Las concentraciones en fase gas de 2,5-DMF (15000 ppm) y de 2-MF (18000 ppm) se obtienen con ayuda de una bomba isocrática HPLC y una línea calefactada. El hollín se recolecta en un cartucho de fibra de cuarzo, al final del sistema de reacción. Los $\mathrm{PAH}$ formados en la fase gas se capturan en la resina XAD-2, la cual se coloca después del recolector de hollín. Las paredes del reactor se lavan con diclorometano para recolectar los PAH adheridos. Se usa un cromatógrafo de gases, para analizar hidrocarburos ligeros. Al finalizar el experimento, las muestras de hollín, de las paredes del reactor y de la resina, se someten a una extracción Soxhlet, y el extracto obtenido se concentra usando un rotavapor. Posteriormente, la identificación y cuantificación de los PAH se lleva a cabo usando un cromatógrafo de gases acoplado a un espectrómetro de masas (GC-MS). El método utilizado para la cuantificación de los PAH fue desarrollado por nuestro grupo de investigación en un trabajo anterior [4].

\section{Resultados}

Se ha analizado el rendimiento a hollín, gases ligeros y $\mathrm{PAH}$, en relación al total de carbono que entra en el sistema de reacción.

En la Figura 1, se puede observar que, para los dos compuestos analizados, el rendimiento a hollín incrementa al aumentar la temperatura de reacción, mientras que disminuye el rendimiento a gases y PAH. Además, los porcentajes de hollín, gases y $\mathrm{PAH}$ obtenidos son similares para ambos 
compuestos, aunque ligeramente mayores para 2,5DMF.

De la misma manera, se puede observar, en las Figuras 2a y 2b, una similitud para 2,5-DMF y 2MF, en la cuantificación de los 16 PAH, para las tres temperaturas probadas, con concentraciones ligeramente superiores de la mayoría de los $\mathrm{PAH}$ en el caso del 2,5-DMF. Esta diferencia se puede atribuir a la mayor relación C/O presente en el 2,5DMF en comparación con el 2-MF.

\section{Conclusiones}

La formación de hollín es mayor para 2,5-DMF que para 2-MF, en todas las temperaturas probadas, al igual que la formación de PAH.

Para todas las temperaturas estudiadas, y para los dos compuestos analizados, los PAH con 2-4 anillos: NAPH, ACNY, FLUO, PHEN, ANTH, FANTH, y PYR, han sido encontrados en altas concentraciones excepto el ACN, presente en concentraciones bajas. Los $\mathrm{PAH}$ con alto peso molecular: B(a)A, CHR, B(b)F, B(k)F, B(a)P, I(123-cd)P, DB(ah)A, y B(ghi)P, han sido detectados en menores concentraciones.

Los resultados obtenidos de la formación de hollín, para los dos compuestos estudiados, podrían determinar su posible selección como combustible renovable.

\section{Agradecimientos}

Los autores agradecen a MINECO y FEDER (Proyecto CTQ2012-34423) y al Gobierno de Aragón por la financiación recibida. Fausto Viteri agradece a la Secretaría Nacional de Educación Superior, Ciencia, Tecnología e Innovación (SENESCYT) de Ecuador, la beca predoctoral otorgada.

\section{Referencias}

[1] ROMÁN-LESHKOV, Y., BARRETT, C. J., LIU, Z. Y., and DUMESIC, J. A. Production of dimethylfuran for liquid fuels from biomass-derived carbohydrates. Nature. 2007, 447, 982-986.

[2] TRAN, L.S., SIRJEAN, B., GLAUDE, P. A., FOURNET, R., and BATTIN-LECLERC, F. Progress in detailed kinetic modeling of the combustion of oxygenated components of biofuels. Energy. 2012, 43 (1), 4-18.

[3] RUIZ, M. P., CALlEJAS, A., MILlERA, Á., ALZUETA, M. U., and BILBAO, R. Soot formation from $\mathrm{C}_{2} \mathrm{H}_{2}$ and $\mathrm{C}_{2} \mathrm{H}_{4}$ pyrolysis at different temperatures. Journal of Analytical and Applied Pyrolysis. 2007, 79 (12), 244-251.

[4] SÁNCHEZ, N., SALAFRANCA, J., CALLEJAS, A., MILLERA, Á, BILBAO, R., and ALZUETA, M. U. Quantification of polycyclic aromatic hydrocarbons (PAHs) found in gas and particle phases from pyrolytic processes using gas chromatography-mass spectrometry (GC-MS). Fuel. 2013, 107, 246-253.

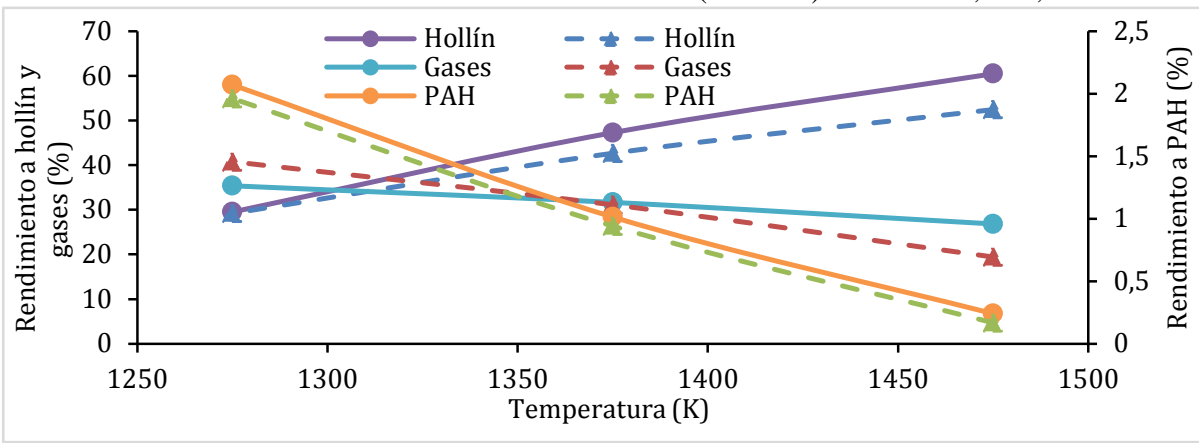

Figura 1. Influencia de la temperatura en el rendimiento a hollín, gases y PAH. Las líneas continuas representan los experimentos con 2,5-DMF y las líneas discontinuas representan los experimentos con 2-MF.
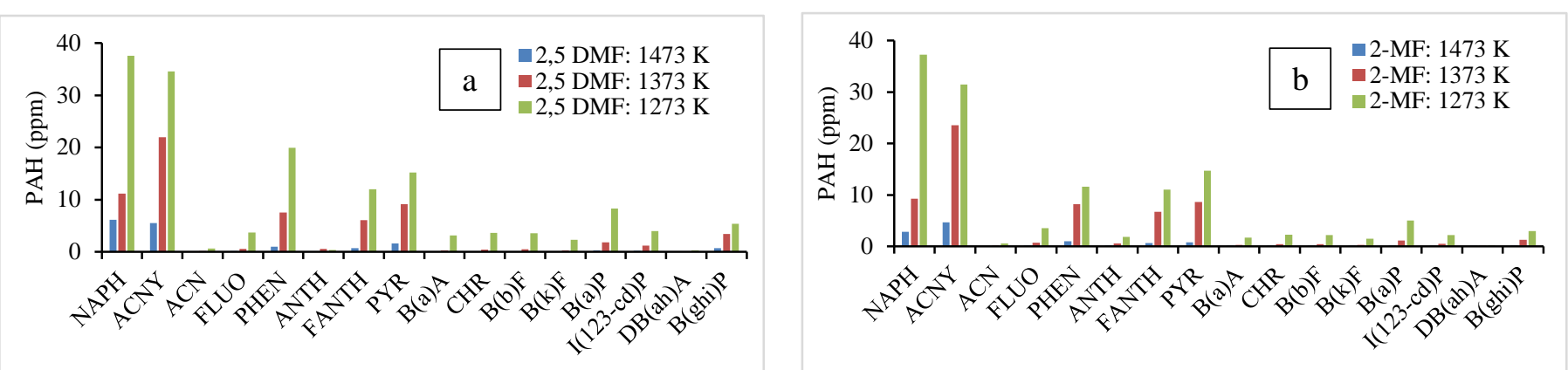

Figura 2. Concentraciones de los 16 PAH a diferentes temperaturas de reacción: (a) 2,5-DMF, (b) 2-MF 